\title{
Tools \& Techniques of Failure Analysis
}

\author{
Michael E. Stevenson
}

Published online: 20 May 2016

(C) ASM International 2016

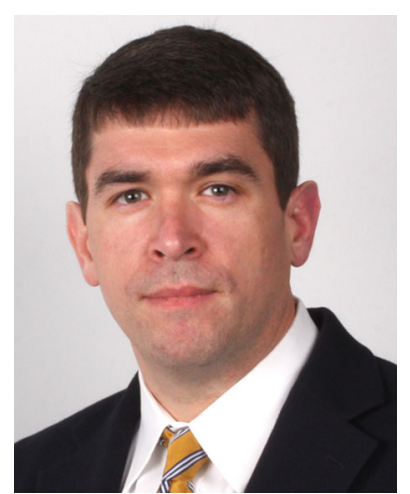

Beginning with this issue of JFAP, you will notice a new type of article that the Editorial Board hopes will become a staple for our readership: Tools \& Techniques of Failure Analysis. This type of article is intended to be a rapid communication of particular methodologies that are employed in failure investigations, and to encourage practitioners to publish these techniques in order to advance the state of the art.

At its foundations, the Tools \& Techniques concept was pioneered by Chuck Morin, a longstanding leader and one of the founders of the ASM Failure Analysis Committee. Chuck's vision was that at our annual meetings, we dedicate a session to the presentation of the current state of the art methodologies in Failure Analysis practice. Since that first year of the session, Tools \& Techniques has been one of the best attended and best prepared sessions each year, attracting failure analysis professionals from all over the world to present the latest methodologies and hardware being utilized in failure investigations. With Chuck's untimely passing in 2008, the Failure Analysis Committee of ASM carried the session he championed forward as an annual standing session at our meetings.

2016 is a landmark year for failure analysis, with the evolution of the ASM Failure Analysis Committee into the Failure Analysis Society (FAS), a standalone affiliate society of ASM International. Similarly, we intend for the inclusion of the Tools \& Techniques manuscripts in the Journal to become a popular and critical vehicle for the transaction of knowledge in our field.

Tools \& Techniques manuscripts are intended to be shorter form, communication-styled manuscripts that focus on the execution and applicability of relatively new or novel methods as applied in practical failure investigations. Details regarding specific case histories are not required, allowing investigators to focus on their methods rather than specific aspects of an investigation that may be confidential or otherwise difficult to publish. We hope that this style manuscript will enable a faster and more extensive dissemination of the tools of our trade, and expand the pool of potential authors in JFAP. These manuscripts will still be fully peer reviewed, as are our regular and case history manuscripts.

We look forward to your continued contributions. 